\title{
Tratamento nutricional da bulimia nervosa
}

\author{
Nutritional therapy for bulimia nervosa
}

Marle dos Santos ALVARENGA'

Fernanda Baeza SCAGLIUSI²

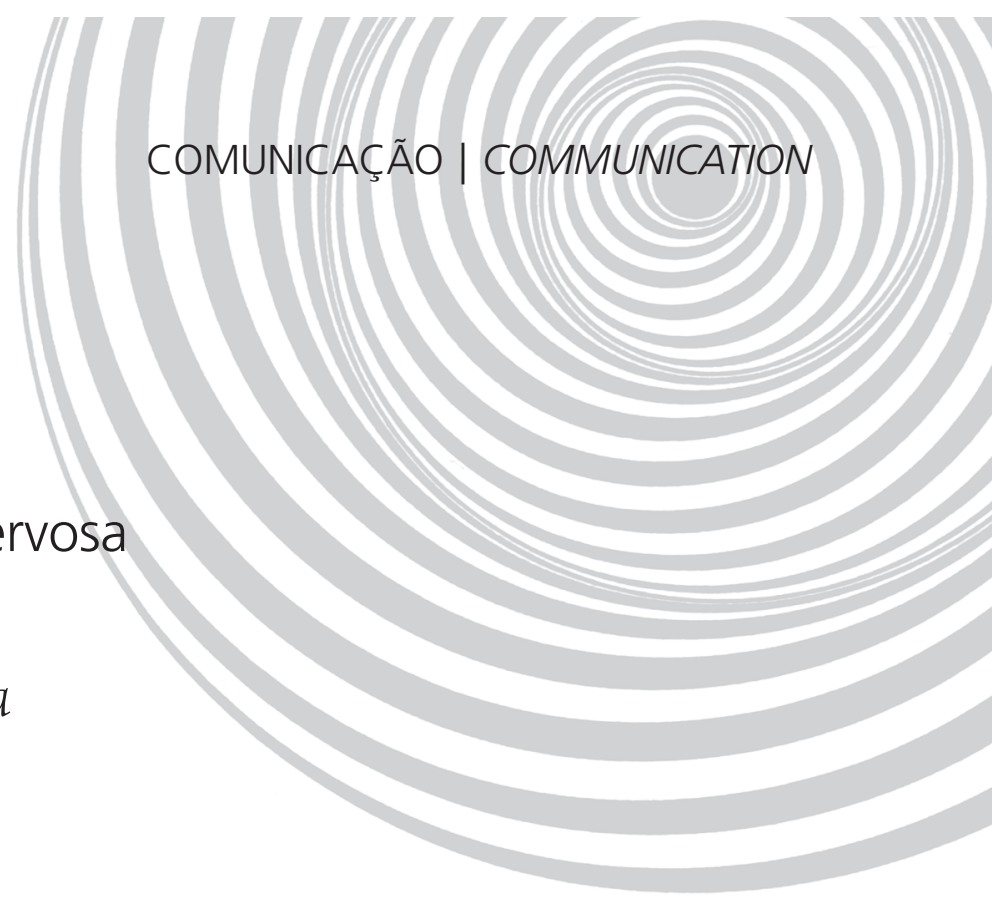

RE S U M O

A bulimia nervosa é um transtorno alimentar caracterizado por compulsões alimentares e métodos compensatórios recorrentes. Os pacientes apresentam ingestão alimentar inadequada e comportamentos alimentares disfuncionais. O adequado tratamento do transtorno requer uma equipe multiprofissional e terapia nutricional especializada. Compreender as características desse transtorno, os padrões de consumo e o comportamento alimentar, bem como atentar para as atitudes alimentares dos pacientes, é fundamental para o planejamento e para a adequada condução da abordagem nutricional. A terapia nutricional para esse transtorno é diferenciada, exigindo do nutricionista maiores habilidades de aconselhamento nutricional. Educação nutricional e aconselhamento nutricional, com ênfase na abordagem de atitudes alimentares e insatisfação corporal, são o foco da terapia nutricional. Para o atendimento eficaz desses pacientes e o sucesso no tratamento nutricional, é importante que o profissional se mantenha atualizado sobre nutrição e transtornos alimentares e procure especialização e experiência nessa área do conhecimento.

Termos de indexação: Aconselhamento. Bulimia nervosa. Hábitos alimentares. Terapia nutricional.

A B S T R A C T

Bulimia nervosa is an eating disorder characterized by binge eating and compensatory behaviors. The patients present inappropriate food intake and dysfunctional eating behaviors. Proper treatment of this disorder requires a multidisciplinary team and specialized nutrition therapy. It is fundamental to understand the characteristics of this disorder, the intake patterns and the eating behavior, and be attentive to the eating attitudes of these patients to plan and conduct a nutritional approach properly. The nutrition therapy for this disorder is specific and demands greater skillfulness in nutrition counseling from the dietician. Nutrition therapy focuses on nutrition education and nutrition counseling, mainly addressing eating attitudes and dissatisfaction with body image. The professional must keep abreast on nutrition and eating disorders and seek specialization and experience in this area of knowledge to provide efficient care for these patients and succeed in their treatment.

Indexing terms: Counseling. Bulimia nervosa. Food habits. Nutrition therapy.

\footnotetext{
1 Universidade de São Paulo, Faculdade de Medicina, Instituto de Psiquiatria, Ambulatório de Bulimia e Transtornos Alimentares. R. Ovídio Pires de Campos, 785, 05403-010, São Paulo, SP, Brasil. Correspondência para/Correspondence to: M.S. ALVARENGA E-mail: <marlealv@uol.com.br>.

2 Universidade Federal de São Paulo, Departamento de Ciências da Saúde. Santos, SP, Brasil.
} 


\section{N T R O D U Ç Ã O}

A Bulimia Nervosa (BN) é um Transtorno Alimentar (TA) reconhecido desde sua descrição por Gerald Russell em $1979^{1}$ e publicação no DSM-III em 1980². Atualmente, os critérios diagnósticos são definidos pela Associação de Psiquiatria Americana no DSM-IV³ e pela Organização Mundial de Saúde no CID-104.

Assim como os demais quadros de TA, os aspectos nutricionais são centrais na BN e devem ser adequadamente compreendidos para que se ofereça o tratamento adequado. O objetivo desta comunicação é discorrer sobre esses aspectos nutricionais dos pacientes com BN e discutir pontos fundamentais para o tratamento nutricional, apresentando uma proposta de tratamento de acordo com as diretrizes atuais e com a experiência de centros de referência nacional para estudo e tratamento desses transtornos.

Embora o objetivo do trabalho não seja uma revisão sistemática, a revisão da literatura foi realizada nas bases PubMed, Lilacs e SciELO a partir de 1980 (ano da definição do critério diagnóstico de BN) até 2008, buscando-se por palavra e utilizando-se os unitermos "bulimia nervosa", "tratamento" e "nutrição". Considerando as três bases, apenas onze artigos sobre tratamento nutricional da $B N$ foram encontrados, e nenhum descrevia detalhadamente as intervenções realizadas. Como a presente comunicação visa a apresentar as técnicas de tratamento nutricional, textos específicos sobre o tema tiveram de ser consultados, já que descrevem tais técnicas detalhadamente, ao contrário dos artigos. Artigos sobre padrão, consumo e comportamento alimentar na BN foram também levantados, a partir de 1980 até 2008, para descrição da alimentação desses pacientes.

\section{Aspectos nutricionais dos indivíduos com bulimia nervosa}

A bulimia nervosa é um quadro clínico no qual a estrutura, consumo e atitudes alimentares estão alterados. A estrutura alimentar é um termo que pode ser aplicado aos horários, tipo e regularidade das refeições; já o consumo alimentar é um conceito que envolve a ingestão energética e de macro e micronutrientes. Atitude alimentar parece ser a melhor expressão para abarcar as diversas formas de relacionamento entre $\mathrm{o}$ indivíduo e os alimentos.

De forma geral, atitude pode ser definida como "uma organização duradoura de crenças e cognições em geral, dotada de carga afetiva pró ou contra um objeto definido, que predispõe a uma ação coerente com as cognições e crenças relativas a este objeto" ${ }^{5}$. Esse conceito possui três componentes: o cognitivo (conhecimento e crenças que o indivíduo tem acerca do objeto atitudinal), o afetivo (sentimentos favoráveis ou desfavoráveis ao objeto atitudinal) e o comportamental (predisposição para agir de forma coerente com os componentes cognitivo e afetivo). Assim, se um indivíduo tem uma atitude favorável a um objeto, pode aceitá-lo; se a atitude é negativa, pode evitá-lo. Desse modo, atitudes alimentares são definidas como crenças, pensamentos, sentimentos e comportamentos para com os alimentos $^{6}$. As atitudes alimentares não são fenômenos inteiramente racionais e individuais, pois sofrem influências subjetivas, sociais, ambientais e culturais.

A BN é caracterizada por um ciclo constituído por dieta, compulsão e purgação, com padrão alimentar descrito como "caótico e bizarro". A restrição tem papel fundamental no início e perpetuação do quadro. Assim, a compulsão pode ser desencadeada pela restrição e por fatores emocionais; e a purgação é usada pelos pacientes com o objetivo de eliminar o excesso ingerido, trazer sensação de alívio, de "purificação" e catarse?.

O padrão alimentar é muito caótico e, portanto, a ingestão de energia e nutrientes vai depender da fase; restritiva ou compensatória. Encontra-se então grande variabilidade na ingestão alimentar, com refeições também muito irregulares. Dessa forma, diversos estudos encon- 
traram grande variação na ingestão de energia entre pacientes com BN. Mitchell \& Laine ${ }^{8}$, estudando a ingestão diária no segundo dia de internação, encontraram média de $451 \mathrm{kcal}$ (com variação de 69 -10 620kcal). Já Hetherington et al. ${ }^{9}$, em estudo de laboratório, encontraram média de 9,378 (desvio-padrão de 1,143)kcal/dia. Os estudos realizados no Brasil mostram também extrema variabilidade na ingestão de energia. Um estudo transversal que avaliou 15 dias de diário alimentar de 30 pacientes com BN em tratamento no Ambulatório de Bulimia e Transtornos Alimentares (AMBULIM) do Instituto de Psiquiatria do Hospital das Clínicas da Universidade de São Paulo encontrou média de $2201 \mathrm{kcal}$ (com variação de 158 a $19254 \mathrm{kcal})^{10}$. Já um estudo de seguimento do mesmo serviço encontrou variação de 337 a 4 094kcal/dia, para as pacientes antes do início do tratamento multiprofissional, e variação de 641 a $2131 \mathrm{kcal} / \mathrm{dia}$ após seis meses de tratamento ${ }^{11}$.

Similarmente, a ingestão de macronutrientes (como contribuição percentual para o valor energético total) é também muito variada; Mitchell \& Laine ${ }^{8}$ encontraram ingestão média de $49 \%$ de carboidratos, $8,0 \%$ de proteínas e $43,0 \%$ de lipídeos e Woell et al. ${ }^{12}$ observaram ingestão média de $41,0 \%$ de carboidratos, $14,0 \%$ de proteínas e 38,0\% de lipídeos.

O estudo transversal brasileiro supracitado encontrou ingestão média de $53,2 \%$ de carboidratos, $16,8 \%$ de proteínas e $30,7 \%$ de lipídeos $^{10}$. O estudo de seguimento brasileiro citado anteriormente encontrou variação de 40,3\% a $72,3 \%$ na ingestão de carboidratos; 9,9\% a 20,8\% na ingestão de proteínas e 18,8\% a 43,2\% na ingestão de lipídeos antes do início do tratamento multiprofissional. Após seis meses de tratamento, os seguintes intervalos foram encontrados: de $40,3 \%$ a $60,2 \%$ no consumo de carboidratos, $12,6 \%$ a $20,6 \%$ no consumo de proteínas e $21,8 \%$ a $42,3 \%$ no consumo de lipídeos ${ }^{11}$.

As compulsões alimentares são centrais no transtorno. A American Psychiatry Association ${ }^{3}$ define uma compulsão como "comer, dentro de um período restrito de tempo (por exemplo, até duas horas), uma quantidade de comida muito maior do que outra pessoa semelhante comeria no mesmo período de tempo e nas mesmas circunstâncias, com a sensação de perda de controle". Classicamente, os alimentos consumidos nesses episódios são aqueles considerados proibidos durante as refeições não compulsivas. Alguns estudos encontraram entre os alimentos mais frequentes dos episódios biscoitos, doces em geral, massas, pães e líquidos em grande quantidade (utilizados para diluir o conteúdo estomacal)9,10.

A relação entre as restrições alimentares e as compulsões deve ser também adequadamente compreendida. Trabalho de revisão de Polivy ${ }^{13}$ discute os principais mecanismos desse fenômeno: a maioria das pacientes com BN afirma que primeiro realizaram dieta e que as compulsões vieram depois. Além disso, muitas pacientes com anorexia nervosa (que praticam extrema restrição alimentar) desenvolvem compulsões e algumas evoluem para a BN. Assim, pode-se dizer que a restrição alimentar é característica essencial do transtorno alimentar e constitui seu passo inicial. Ela é condição necessária para o TA, mas não suficiente, já que interage com outros fatores etiológicos. Assim, embora a opinião leiga tenda a considerar que aqueles que têm compulsões alimentares devam fazer restrições alimentares depois, para compensar, existem evidências de que as dietas ocasionam a compulsão. Por exemplo, animais submetidos à restrição passam a ingerir mais alimentos quando retornam ao peso normal - especialmente alimentos mais saborosos ${ }^{14,15}$. No estudo de Polivy ${ }^{13}$, voluntários eutróficos (homens) se submeteram à restrição alimentar por seis meses até perderem $25 \%$ do peso corporal. Diversas consequências da restrição foram notadas, entre elas o desenvolvimento de compulsão alimentar após o experimento.

Esse resultado não foi encontrado apenas nesse tipo de situação extrema. Um estudo longitudinal com amostra representativa de adolescentes observou que fazer dietas drásticas aumen- 
ta 18 vezes o risco de desenvolvimento de TA, enquanto fazer dietas moderadas aumenta cinco vezes $^{16}$. Uma explicação fisiológica para isso seria o fato de as dietas levarem a um peso abaixo do set-point e que a compulsão restauraria o peso adequado. Para tanto, haveria aumento da fome fisiológica, com aumento dos sinais de fome enviados pelo sistema nervoso central. Esse aumento é, de fato, verificado em quem faz dieta, mas nem sempre há a presença de compulsão. Além disso, a compulsão gerada pela restrição também é vista em sujeitos que não perderam muito peso e/ou não fizeram restrições extremas. Assim, deve existir um mecanismo psicológico que gere a compulsão após a restrição ${ }^{17}$.

Fazer dieta aumenta a preocupação em relação aos alimentos e frequentemente os indivíduos dividem os alimentos em bons e ruins. A dieta também requer que o indivíduo ignore suas vontades e as pressões internas (fisiológicas) para regulação do peso corporal, aumentando o conflito entre o sujeito e a comida e tornando-o cada vez mais dependente de controles cognitivos severos. Qualquer acontecimento que quebre esse controle (como frustração e stress) pode acarretar uma compulsão. É importante lembrar que a dieta substitui o controle interno da ingestão (fome/ saciedade) por um controle externo, planejado e determinado cognitivamente, o que pode causar desregulação no controle normal da ingestão ${ }^{17}$. Esses controles externos também causam frustração pela negação dos alimentos favoritos e estresse por constantemente se opor a uma necessidade biológica.

As compulsões são muitas vezes também desencadeadas pela visão perfeccionista que os indivíduos com BN têm do que é alimentação adequada. Por considerarem apenas a alimentação restritiva como saudável, os pacientes não se permitem comer alguns alimentos que podem não primar pelo valor nutricional, mas que proporcionam muito prazer, como o chocolate, por exemplo. Assim, no momento em que comem esses alimentos, sentem uma culpa muito grande, seguida por um pensamento dicotômico que prega "já que eu comi um, comerei vários, pois a partir de amanhã nunca mais comerei esse alimento". Esse modelo foi chamado por Kausman ${ }^{18}$ de "a mente de quem faz dieta" e exemplifica como o pensamento restritivo pode desencadear compulsões, independentemente dos efeitos fisiológicos da restrição. Esse mecanismo foi também demonstrado em estudos experimentais nos quais os sujeitos recebem um alimento (chamado de preload) antes de realizarem a ingestão de uma refeição. Quando o preload é altamente energético, indivíduos que não restringem sua alimentação comem menos na próxima refeição, enquanto as pessoas que fazem dietas comem mais. O mesmo ocorre quando o sujeito apenas acha que o preload é altamente energético. O indivíduo que faz dieta não regula sua ingestão pela fome e saciedade e sim por uma suposta "moral" que o permite ou não comer. Ao quebrar essa regra moral, "aproveita" aquele momento para "cometer seu último crime", antes de receber o castigo dietético novamente.

A decisão prévia de purgar também pode desencadear compulsões e os episódios são algumas vezes planejados. Porém, essa mesma regra rígida de "alimentação saudável" pode fazer com que os pacientes considerem qualquer ingestão de um alimento "proibido" compulsiva, independentemente da quantidade consumida. Woell et al. ${ }^{12}$ descrevem a situação afirmando que "o que os pacientes chamam de episódio bulímico pode depender da quantidade bem como do conteúdo energético do alimento consumido e ainda da disposição psicológica. Quando pensam ter comido demais um tipo especial de alimento, já se sentem culpados. Pode haver uma ideia subjetiva de haver comido muito ou consumido algum tipo especial de alimento. Usualmente os pacientes preferem os alimentos mais disponíveis, de acesso irrestrito, que não necessitam ser cozidos nem preparados e que possam ser purgados facilmente em grandes quantidades".

Estudando o que os pacientes com BN chamavam de compulsão em um estudo que utilizou a metodologia do discurso do sujeito coletivo, 
Romano \& Philippi ${ }^{19}$ encontraram que os estímulos citados como desencadeadores foram questões emocionais - ansiedade, tédio, irritabilidade, culpa, frustração, mau humor, raiva -, consumo de algo considerado "proibido ou inadequado", consumo de grandes quantidades de alimentos, pressão social e cobrança por corpo mais magro e incerteza sobre o que comer depois.

Estudo transversal com pacientes com BN no Brasil ${ }^{10}$ encontrou que os episódios bulímicos foram mais frequentes na sequência das refeições principais - almoço e jantar - ou seja, as pacientes começavam uma refeição "normal" e acabavam por aumentar a quantidade dos alimentos em geral do almoço ou do jantar e na sequência consumiam doces e outros. Assim, alimentos típicos da dieta brasileira (arroz, feijão, carne) e alimentos densos em energia (doces, biscoitos, sorvetes) estiveram presentes. Os episódios bulímicos também foram mais frequentes no fim da tarde e começo da noite, horários associados a uma maior privacidade e acesso aos alimentos por coincidirem com retorno para casa da escola ou trabalho. A duração dos episódios foi menor que 30 minutos em $75,9 \%$ dos casos e os vômitos foram provocados em até 15 minutos após a ingestão em $41,4 \%$ dos casos.

Essas inadequações de padrão e consumo alimentar não são únicas de pacientes com BN, pois mesmo sem $T A$, indivíduos podem apresentar padrões desbalanceados de alimentação e inadequações de consumo de energia e nutrientes. São as atitudes alimentares que são o grande diferencial e que são típicas do transtorno. As restrições alimentares são persistentes, os pacientes insistem em fazer "dieta", acreditando que dessa forma podem perder peso ou até controlar seus episódios compulsivos - quando é exatamente o contrário. Existe também uma visão dicotômica dos alimentos, um pensamento "tudo ou nada"; quando acreditam que falharam ou erraram, muitas vezes os pacientes desistem de qualquer tentativa de controle e adequação, "optando" por um excesso de alimentos ainda maior ${ }^{20}$.

Os pacientes com BN ainda expressam repugnância pelos alimentos, afirmando terem raiva de sentir fome, e ódio pela existência da comida; e relatam uma relação de culpa, repulsa e incompetência para lidar com o alimento. Assim, negam muitas vezes suas necessidades fisiológicas, como se comer fosse uma opção e não uma necessidade; afirmam frequentemente não suportar a sensação do alimento dentro do estômago, gostando da sensação de estômago vazio, preferindo, portanto, às vezes, os alimentos líquidos por sentirem que são digeridos mais facilmente ${ }^{20}$.

Os pacientes apresentam muita dificuldade em selecionar o que comer e têm um "comer social" prejudicado pelo medo de comer os alimentos que julgam "perigosos", e, de fato, podem ter perda de controle em situações com alimento em abundância. Buscam também o alimento para resolver problemas e compensar frustrações ${ }^{12}$. Indivíduos com BN também apresentam uma série de cognições errôneas sobre conceitos nutricionais, mitos e crenças distorcidas sobre alimentos e alimentação. Preocupam-se permanentemente com o peso, chegando a pesar-se inúmeras vezes ao dia: antes e depois de comer, antes e depois de vomitar, antes e depois de ir ao banheiro.

As inadequações de estrutura e de consumo alimentar devem ser tratadas, mas essas atitudes disfuncionais exigem um tratamento nutricional diferenciado, que acesse as cognições errôneas e que permita mudança de comportamento, crença e sentimento.

\section{Tratamento nutricional}

O tratamento nutricional para $\mathrm{BN}$, assim como para Anorexia Nervosa (AN), é um trabalho diferenciado das abordagens nutricionais para outras doenças. Do mesmo modo que, em outros tratamentos, o bom estado nutricional é objetivo básico a ser alcançado, outros aspectos devem também ser considerados numa visão mais ampla sobre a mudança de atitudes dos pacientes para com os alimentos e a nutrição. Dessa forma, considera-se que o nutricionista que trabalha com TA deva ser chamado de terapeuta nutricional|21,22, 
que é um termo que engloba todas essas ações diferenciadas perante esses pacientes.

A American Dietetic Association ${ }^{22,23}$ estabeleceu diretrizes básicas para o tratamento nutricional dos transtornos alimentares, propondo uma fase inicial baseada em educação nutricional e uma fase seguinte baseada em aconselhamento para mudar comportamentos e atitudes alimentares.

A educação nutricional proposta para esses pacientes englobará tópicos presentes em programas de nutrição clássicos, como alimentação balanceada, pirâmide dos alimentos, necessidades nutricionais. O diferencial é o foco: os mitos e crenças a serem trabalhados e desconstruídos durante o tratamento ${ }^{24}$.

Já o aconselhamento nutricional é uma abordagem muito específica, na qual técnicas cognitivo-comportamentais, interacionais, relacionais, educacionais e de entrevista motivacional são utilizadas para acessar crenças, sentimentos e comportamentos disfuncionais dos pacientes e para ajudá-los numa jornada de mudanças. Ambas as fases de tratamento exigem um profissional treinado, com experiência em transtornos alimentares. Segundo Reiff \& Reiff ${ }^{7}$, não se trata apenas de aprender técnicas; muitas vezes o terapeuta nutricional deve "re-aprender nutrição", abandonando a abordagem restritiva e lipofóbica que domina a ciência da nutrição e construindo um novo saber no qual a alimentação não é entendida apenas como um meio destinado a atingir um fim.

A principal meta do tratamento é aprender a comer de modo "normal", o que não é simples para pacientes que acreditam ter amplos conhecimentos sobre alimentação. Na maioria das vezes, entretanto, os pacientes têm apenas muito conhecimento sobre o valor energético dos alimentos e interpretam tais dados de maneira rígida e enviesada. Assim, alguns dizem nunca saber o que devem comer. $\mathrm{O}$ tratamento nutricional especializado para TA deve ser planejado de modo a ajudar os pacientes a entender e serem aptos a colocar os alimentos e os nutrientes numa perspectiva apropriada $^{23}$.
O terapeuta nutricional tem parte importante no tratamento dos TA e é essencial na equipe multiprofissional; é o único qualificado profissionalmente para prover o tratamento nutricional especializado para esses pacientes 22,25 .

\section{Programa educacional}

O programa educacional para BN visa, basicamente, a promover orientação alimentar para o estabelecimento de padrões nutricionais adequados. Os objetivos principais são: a eliminação do ciclo "dieta - compulsão - purgação" e o estabelecimento de um padrão alimentar adequado.

Os terapeutas nutricionais do AMBULIM desenvolveram, a partir de sua experiência e da literatura científica, um programa de tratamento básico de 18 semanas $^{24}$. Os tópicos desenvolvidos em cada semana são: 1) Como é o tratamento nutricional: métodos e importância; 2) O que é bulimia nervosa? 3) O papel da restrição alimentar na bulimia nervosa; 4) Definição dos nutrientes: o que são e para que servem; 5) Vitaminas e minerais: fontes e importância; 6) A roda de alimentos: porque preciso de todos nutrientes?; 7) Pirâmide de alimentos: como balancear uma refeição? 8) Como variar a alimentação? 9) Conceitos de fome, apetite e saciedade; 10) Fibras e funcionamento do intestino; 11) Necessidades energéticas e metabolismo basal; 12) Gasto energético e atividade física; 13) Absorção e digestão dos alimentos; 14) Dietas: porque não funcionam; 15) Peso adequado, índice de massa corporal e compleição física; 16) Alimento diet e light; 17) Como comer em situações sociais? 18) Avaliação do período e discussão de técnicas para manutenção de hábitos saudáveis.

Além de explicar o programa e sua importância, o tratamento é iniciado com a compreensão, por parte do paciente, do comportamento bulímico e suas alterações em relação ao comportamento normal.

As abordagens semanais possuem uma série de diferenciais em relação a outros progra- 
mas de educação nutricional. Nas semanas 2, 3 e 14 , o foco é apresentar a restrição alimentar como iniciadora e perpetuadora do ciclo da BN. Durante todo programa é preciso educar os pacientes a planejar e a realizar uma alimentação saudável e atentar para que não troquem uma "dieta restritiva desbalanceada" por uma "dieta restritiva balanceada".

As semanas que discutem conceitos nutricionais visam a desmistificar o medo que esses pacientes têm de ingerir, especialmente, gorduras e carboidratos, e ainda mostrar que alimentos que são considerados "perigosamente engordativos" - carnes vermelhas e ovos - são também muito ricos em nutrientes.

Quando se discute metabolismo, atividade física e gasto energético, é preciso desfazer a ideia de "quanto menos calorias se ingerir, melhor", bem como diferenciar os usos da atividade física: saúde, purgação, compulsão ${ }^{26}$.

Na sessão sobre peso adequado, índice de massa corporal e compleição física, deve-se desfazer a ideia de que o corpo é totalmente maleável, bem como diferenciar o peso "socialmente desejável" do peso possível e saudável e associar o peso à saúde e não à estética ou ao valor pessoal.

As sessões podem ser feitas em grupo, no caso de ambulatórios e hospitais, ou individualmente em consultórios. As sessões em grupo são um fórum interessante para que o paciente, ao perceber que outros também têm os mesmos medos e angústias, não se sinta isolado em seu problema e para que soluções possam ser discutidas. Porém, alguns cuidados devem ser tomados quando tais grupos são conduzidos, pois alguns pacientes, especialmente aqueles que também apresentam transtornos de personalidade, tendem a competir para mobilizar mais atenção do grupo e dos profissionais. Os terapeutas nutricionais devem estar atentos a tais situações e procurar supervisão de psiquiatras e/ou psicólogos da equipe.

Para trabalhar questões mais específicas de cada um dos pacientes, o instrumento de esco-
Iha é o diário alimentar. No diário são registrados a data, o horário, o local das refeições, os tipos e a quantidade de alimentos ingeridos, além da ocorrência de episódios de compulsão alimentar e purgações. Outras observações podem também ser feitas, como dar uma nota para a fome, apontar se ficou satisfeito, relatar com quem fez as refeições e quais as sensações e sentimentos associados àquele momento. $\mathrm{O}$ uso do diário alimentar é prática recomendada e de bom resultado no tratamento dos TA, sendo considerado uma técnica comportamental de automonitoração que provê controle, disciplina e avaliação constantes. O diário pode ainda simbolizar a relação entre o terapeuta nutricional e o paciente, uma vez que é um modo do paciente ter a "presença" do terapeuta nutricional consigo ao longo da semana ou período, tornando-se um documento pessoal que aponta a evolução do pro$\operatorname{cesso}^{26,27}$.

\section{Aconselhamento nutricional}

O aconselhamento nutricional não é uma fase isolada do tratamento, mas sim uma abordagem a ser aplicada desde o início e também durante o programa educacional. Para atender as necessidades e trabalhar os medos e crenças de cada um, o aconselhamento deve ser um trabalho individual.

O aconselhamento nutricional foca o desenvolvimento de uma relação colaborativa com os pacientes, que deve ser mantida para que haja informação e intervenções corretas. O aconselhamento nutricional para os TA é uma técnica especializada que foca a correção dos comportamentos e crenças alteradas nas áreas de alimentação, corpo e atividade física. $\mathrm{O}$ aconselhamento nutricional esclarece os antecedentes dos comportamentos alimentares, provê informação nutricional, dá suporte para experimentar novos comportamentos e avalia os resultados ${ }^{27}$.

O principal objetivo do aconselhamento nutricional é a troca de padrões alimentares alterados e não saudáveis por padrões saudáveis e 
organizados. O aconselhamento nutricional também foca a restauração e o monitoramento do peso corporal ${ }^{27,28}$. Esse trabalho é mais complexo porque os pacientes muitas vezes se acham autossuficientes e resistem à ajuda profissional. Ter uma postura de suporte em vez de uma postura de confronto é fundamental. A motivação do paciente para a recuperação aumenta quando o terapeuta nutricional reconhece a dificuldade de mudar comportamentos alimentares alterados e simpatiza com o estresse do paciente no caminho da mudança ${ }^{28}$.

Aponta-se que o terapeuta nutricional que atua com TA deve ter uma postura confiante e confortável, ser um ouvinte empático e um colaborador que provê suporte e que tem uma preocupação não intrusiva. Estabelecer uma relação colaborativa requer um terapeuta nutricional ativo, direto, simpático, bem-humorado, honesto e capaz de estabelecer empatia e credibilidade. Também se deve evitar ter uma atitude patriarcal ou maternal perante o paciente ${ }^{27}$.

O terapeuta nutricional deve mostrar interesse genuíno pelas experiências, ansiedades, obsessões e preconceitos dos pacientes. A maioria dos pacientes com TA inicia o tratamento nutricional com certa ambivalência. Uma abordagem curiosa, interessada e empática ajuda pacientes ambivalentes a se sentirem compreendidos. Trabalhar com os preconceitos e os comportamentos mal adaptados dos pacientes com TA pode ser desgastante. Quando o terapeuta nutricional percebe que tem problemas com um paciente específico, ele não deve "culpar" o paciente, mas tentar repensar suas atitudes, procurando entender o que esse paciente desperta ${ }^{27}$.

Trabalhar com os pacientes com TA pode ser especialmente estressante para o terapeuta nutricional devido à centralidade das questões alimentares no quadro e também porque conceitos psicoterapêuticos, como transferência e contratransferência, não são abordados nos cursos de graduação em nutrição.

A transferência e a contratransferência são fenômenos que surgem na relação entre um paciente e um terapeuta, seja este um psicoterapeuta, terapeuta nutricional, psiquiatra ou qualquer outro profissional de saúde. Embora esses sejam conceitos próprios da psicologia e os terapeutas nutricionais não façam (e nem devam fazer) psicoterapia com seus pacientes, caso o terapeuta nutricional não os conheça, ele poderá ser manipulado pelo paciente e até fazer mal a ele ou a si próprio. Assim, terapeutas nutricionais devem procurar especialização, treinamento e supervisão para atender pacientes com TA, pois necessitam entender tais conceitos, aprender mais sobre como lidar com o paciente e manter comunicação com os demais profissionais da equipe multiprofissional ${ }^{22-27}$.

Para que o tratamento tenha sucesso, uma recomendação de aconselhamento é que se explore as metas do paciente. Essa técnica chama-se entrevista motivacional, que é um procedimento centrado no cliente e um método diretivo para aumentar a motivação intrínseca para mudança, explorando e resolvendo as ambivalências $^{29}$. Resolver as ambivalências implica fazer um balanço entre os prós e contras dos comportamentos alimentares alterados, entendendo que os sintomas têm funções na vida dos pacientes, e não só as consequências negativas. Além disso, é preciso fazer um balanço entre os custos das mudanças e os benefícios que as mudanças podem trazer; para tanto se deve entender as motivações individuais ${ }^{29-30}$. Na BN a situação é complexa, pois o paciente deseja parar com suas compulsões, mas são bem menos dispostos a mudar suas estratégias de controle de peso ${ }^{27}$.

Para ajudar o paciente a mudar seus comportamentos é necessário explorar os padrões alimentares e de dieta, e suas razões com o paciente. Quando os aspectos mais e menos positivos são explorados é possível sintetizar e questionar como o paciente se sente. É importante explorar o que o paciente sente sobre mudar para um padrão de alimentação mais saudável ${ }^{27}$.

Stellefson ${ }^{21}$ afirma que a entrevista motivacional permite que o indivíduo reconheça a incompatibilidade do comportamento padrão do 
TA - que tem consequências negativas e perpetua um ciclo de falha e frustração - com funções mais importantes, centrais e de valor para ele, como a relação com sua família, a capacidade de estudo ou trabalho. Se o paciente faz dieta porque acredita que a magreza corresponde a sucesso e atratividade, o terapeuta não deve desqualificar sua opinião e nem tentar mudar diretamente o comportamento em si - fazer dieta. O papel do terapeuta nutricional é ajudar o paciente a entender suas motivações, analisar as crenças envolvidas e os benefícios e prejuízos decorrentes dessa crença e desse comportamento ${ }^{27}$.

\section{Atitudes alimentares}

Uma meta avançada do tratamento é mudar as atitudes dos pacientes para com o alimento e a imagem corporal. Para atingir essa meta, apenas um programa educacional não é suficiente. Além de usar técnicas adequadas de abordagem, novos métodos e sessões devem ser também planejados.

A Terapia Cognitivo-Comportamental (TCC) é apontada como uma boa ferramenta para tratar o comportamento alimentar inadequado e as sensações e sentimentos disfuncionais para com o alimento ${ }^{23}$. Acredita-se que os terapeutas nutricionais devem utilizar técnicas de terapia cognitivo-comportamental - se especializados na abordagem - para trabalhar as atitudes alimentares e não apenas deixar essa tarefa sob responsabilidade única do psicoterapeuta. Segundo a American Dietetic Association ${ }^{22}$, devido ao fato de existirem tantas dietas da moda, falácias sobre alimentação e reforço da mídia sobre conceitos errôneos não é incomum que outros membros da equipe de tratamento - mesmo com experiência em TA - acreditem em crenças alimentares inadequadas. O nutricionista especializado seria, portanto, o único qualificado a prover educação nutricional baseada em ciência ${ }^{22}$.

Observou-se, em estudo realizado com pacientes com BN no Brasil, que após tratamento básico, focado em educação nutricional, os pa- cientes melhoravam em termos de sinais e sintomas, mas não mudavam de modo satisfatório suas atitudes alimentares ${ }^{6,31}$. Acredita-se que mudar atitudes alimentares é mais difícil do que mudar comportamentos e consumo alimentar inadequados.

Assim como se aponta que a mudança efetiva da imagem corporal exige técnicas diferenciadas de abordagem, o tratamento e a abordagem efetivos das atitudes alimentares provavelmente exigem também outras técnicas. Assim, um programa de tratamento para BN que objetive uma melhora da imagem corporal e de atitudes alimentares deve discutir se os padrões de corpo e alimentação apresentados pela sociedade e incorporados pelos pacientes são, de fato, saudáveis, tentando obter como resultado a "legitimização" do prazer, do gosto e da função social dos alimentos. Dinâmicas de grupo utilizadas em outros centros e países são uma boa fonte de referência para elaboração de abordagens terapêuticas diferenciadas ${ }^{32-35}$.

\section{Perspectivas de continuidade das pesquisas na área}

Os TA são mais discutidos e pesquisados atualmente nas áreas médica, psicológica e nutricional. De qualquer forma, no Brasil, as pesquisas com foco nutricional são ainda escassas. Com os unitermos utilizados, apenas seis artigos publicados no Brasil foram encontrados $25,26,36-38$, sendo apenas dois com foco no tratamento nutricional da $\mathrm{BN}^{25,26}$

Na literatura internacional, a busca com esses unitermos resulta em estudos sobre o papel de neurotransmissores e peptídeos ${ }^{39-41}$, tratamentos combinados ${ }^{42-46}$, e alguns com foco específico no tratamento nutricional ${ }^{47-49}$.

Esses trabalhos apontam que o tratamento nutricional deve ser visto como uma primeira intervenção necessária ${ }^{42}$, para todos os pacientes; que a intervenção nutricional é efetiva para BN - com melhoras mantidas em três meses de segui- 
916 | M.S. ALVARENGA \& F.B. SCAGLIUSI

mento- ${ }^{44}$ e que a TCC combinada à terapia nutricional permanece como tratamento de escolha para $\mathrm{BN}^{45}$.

Quanto aos estudos a serem realizados nessa área, sugere-se que a TCC deva ser testada de modo mais intenso para pacientes que apresentaram falhas nos tratamentos com grupo de suporte $^{45}$; que estudos futuros devem avaliar os possíveis efeitos adicionais da TCC e do exercício físico no tratamento da $\mathrm{BN}^{46}$, e que como a BN é um transtorno grave e com baixa taxa de remissão espontânea, o tratamento associado, com abordagem multidimensional e multidisciplinar, é o indicado ${ }^{38}$.

Muitas vezes, de fato, os estudos analisam o efeito do tratamento nutricional isolado (como estratégia única de tratamento) versus a TCC $38,42-46$, mas se acredita que o ideal seria analisar a TCC versus TCC mais tratamento nutricional, uma vez que o tratamento nutricional não deve ser nunca uma estratégia única de tratamento, dada a necessidade de uma equipe e de uma abordagem multiprofissional ${ }^{31}$.

Especificamente na realidade nacional, sugere-se que modelos de tratamento especializado sejam avaliados quanto à eficácia, que novas abordagens sejam testadas - com foco nas mudanças das atitudes alimentares e da relação com o alimento e o corpo -, que intervenções mais prolongadas sejam propostas para se obterem quadros de recuperação mais completos e que se trabalhe a prevenção de recaídas 6,10,11,24-26,31.

\section{O N C L U S Ã O}

Para tratar a estrutura, o consumo e as atitudes alimentares disfuncionais na BN é preciso uma compreensão ampla sobre o significado da alimentação na vida do ser humano: física, social e emocional; e também sobre o modo e as razões pelas quais a alimentação é afetada nesse transtorno alimentar.

Para ser apto para o tratamento nutricional da BN e demais TA, o nutricionista deve aprender técnicas terapêuticas que vão além daquelas específicas de sua profissão e relacionadas à dietoterapia em si, como as técnicas cognitivo-comportamentais, de entrevista motivacional e aconselhamento educacional e comportamental. Deve, ainda, rever seus conceitos sobre o que é verdadeiramente alimentação saudável e também suas crenças pessoais sobre alimentação e peso.

Os tópicos trabalhados nesse tratamento nutricional são específicos e de abordagem diferenciada. A experiência com o modelo exemplificado tem encontrado resultados positivos para melhora dos sintomas bulímicos e de comportamento alimentar ${ }^{11,31}$. Acredita-se que mais pesquisas e esforços conjuntos na avaliação de abordagens nutricionais devam ser feitos para alcançar melhores respostas para a imagem corporal e uma melhor relação dos pacientes para com o alimento.

\section{A GRADECIMENTOS}

À Fundação de Amparo à Pesquisa do Estado de São Paulo (FAPESP) os auxílios que apoiaram o pós-doutorado de Marle Alvarenga (processo 06/56850-9) e de Fernanda Scagliusi (processo 07/59141-1).

\section{COLABORADORES}

Os dois autores contribuíram para revisão do tema e redigiram conjuntamente esta comunicação.

\section{REFERÊ NCIAS}

1. Russell GFM. Bulimia nervosa: an ominous variant of anorexia nervosa. Psychol Med. 1979; 9(3):429-8.

2. American Psychiatric Association. Diagnostic and statistical manual of mental disorders (DSM-III). $3^{\text {rd }}$ ed. Washington (DC): American Psychiatry Association; 1980.

3. American Psychiatry Association. Diagnostic and Statistical Manual of Mental Disorders (DSM-IV). $4^{\text {th }}$ ed. Washington (DC): American Psychiatry Association; 1994.

4. Organização Mundial de Saúde. Classificação de transtornos mentais e de comportamento da CID-10. 
Descrições clínicas e diretrizes diagnósticas. Porto Alegre: Artes Médicas; 1993.

5. Oppenheim AN. Questionnaire design and attitude measurement. New York: Basic Books, 1966.

6. Alvarenga MS, Scagliusi FB, Philippi ST. Changing attitudes, beliefs and feelings towards food in bulimic patients. Arch Latinoam Nutr. 2008; 58(3): 274-9.

7. Reiff DW, Reiff KKL. Eating disorders: nutrition therapy in the recovery process. Maryland: Aspen Publishers; 1992.

8. Mitchell JE, Laine DC. Monitored binge-eating behavior in patients with bulimia. Int J Eat Disord. 1985; 4(2):177-83.

9. Hetherington MM, Altemus $M$, Nelson ML, Bernat AS, Gold PW. Eating behavior in bulimia nervosa: multiple meal analyses. Am J Clin Nutr. 1994; 60(6): 864-73.

10. Alvarenga MS, Negrão AB, Philippi ST. Nutritional aspects of eating episodes followed by vomiting in Brazilian patients with bulimia nervosa. Eat Weight Disord. 2003; 8(2):150-56.

11. Alvarenga MS, Scagliusi FB, Philippi ST. Consumo e padrões alimentares de pacientes com bulimia nervosa antes e depois de tratamento multiprofissional. Rev Bras Nutr Clin. 2004; 19(4):170-7.

12. Woell C, Fichter MM, Pirke KM, Wolfram G. Eating behavior of patients with bulimia nervosa. Int J Eat Disord. 1989; 8(5):557-68.

13. Polivy J. Psychological consequences of food restriction. J Am Diet Assoc. 1996; 96(6):589-92.

14. Boggiano MM, Chandler PC. Binge eating in rats produced by combining dieting with stress. Curr Protoc Neurosci. 2006; 9(9):23A.

15. Hagan MM, Chandler PC, Wauford PK, Rybak RJ, Oswald KD. The role of palatable food and hunger as trigger factors in an animal model of stress induced binge eating. In t J Eat Disord. 2003; 34(2): 183-97.

16. Hetherington MM. Eating disorders: diagnosis, etiology, and prevention. J Nutr. 2000; 16(7-8): 547-551.

17. Polivy J, Herman CP. Etiology of binge eating: psychological mechanisms. In: Fairburn CG, Wilson GT. (Eds.) Binge eating: nature, assessment, and treatment. New York: Guilford Press; 1993.

18. Kausman R. A new perspective to long term weight management. Is there a better way? Aust Fam Physician. 2000; 29(4):303-6.

19. Romano EB, Philippi ST. O que compulsão alimentar? O que é um episódio bulímico? In: Philippi ST, Alvarenga MS. Transtornos alimentares: uma visão nutricional. São Paulo: Manole; 2004.
20. Alvarenga MS, Dunker KLL. Padrão e comportamento alimentar na anorexia e bulimia nervosa. In: Philippi ST, Alvarenga MS. Transtornos alimentares: uma visão nutricional. São Paulo: Manole; 2004.

21. Stellefson E. Winning the war within: nutrition therapy for clients with anorexia or bulimia nervosa. Texas: Helm Publishing; 1999.

22. American Dietetic Association (ADA). Position of the American Dietetic Association: nutritional intervention in the treatment of anorexia nervosa, bulimia nervosa, and eating disorders not otherwise specified (EDNOS). J Am Diet Assoc. 2001; 101(7): 810-19.

23. American Dietetic Association (ADA). Position of the American Dietetic Association: nutritional intervention in the treatment of anorexia nervosa, bulimia nervosa and binge eating. J Am Diet Assoc. 1994; 94(8):902-7.

24. Alvarenga MS, Dunker KLL, Romano ECB, Philippi ST. Terapia nutricional para Transtornos Alimentares. In: Philippi ST, Alvarenga MS. Transtornos alimentares: uma visão nutricional. São Paulo: Manole; 2004.

25. Latterza AR, Dunker KLL, Scagliusi FB, Kemen E. Tratamento nutricional dos transtornos alimentares. Rev Psiq Clin. 2004; 31(4):173-6.

26. Alvarenga MS, Larino MA. Terapia Nutricional na anorexia e bulimia nervosas. Rev Bras Psiquiatr. 2002; 24 (Supl III): 39-43.

27. Herrin M. Nutrition counseling in the treatment of eating disorders. New York: Taylor and Francis; 2003.

28. Rock CL, Curran-Celentano J. Nutritional management of eating disorders. Psych Clin North Am. 1996; 19(4):701-13.

29. Miller WR, Rollnick S. Motivational interviewing: preparing people for change. $2^{\text {nd }}$ ed. New York: Guilford; 2002.

30. Treasure $\mathrm{J}$, Ward A. A practical guide to the use of motivational interviewing in anorexia nervosa. Euro Eat Disord Rev. 1997; 5(2):102-14.

31. Alvarenga MS, Philippi ST. Padrão e comportamento alimentar de bulímicas atendidas no AMBULIM. In: Philippi ST, Alvarenga MS. Transtornos alimentares: uma visão nutricional. São Paulo: Manole; 2004.

32. Cash TF. The body image workbook: an 8-step program for learning to like your looks. Oakland: New Harbinger; 1997.

33. Christian SS. Working with groups to explore food and body connections. Michigan: Whole Person Associates; 1996. 
34. LoBue A, Marcus M. The Don't Diet Live It Workbook. Carlsbad: Gurze; 1999.

35. Waterhouse D. Working as a team to enhance eating habits and self-esteem. Portland: Waterhouse; 1997.

36. Segal A, Cordás TA, D’Elia FLG, Larino MA, Alvarenga MS, Bucaretchi $\mathrm{H}$, et al. Bulimia nervosa II: tratamento. J Bras Psiquiatr. 1995; 44(supl.1): S25-S31.

37. Silva FML, Silva FJL. Bulimia nervosa: um grande desafio. J Bras Med. 1998; 75(2):78-80.

38. Bacaltchuk J, Phillipa H. Tratamento da bulimia nervosa: síntese das evidências. Rev Bras Psiquiatr. 1999; 21(3):184-7.

39. Kaye W, Gendall K, Strober M. Serotonin neuronal function and selective serotonin reuptake inhibitor treatment in anorexia and bulimia nervosa. Biol Psychiatry. 1998; 44(9):825-38.

40. Monteleone $P$, Martiadis $V$, Rigamonti $A E$, Fabrazzo M, Giordani C, Muller EE, et al. Investigation of peptide $Y Y$ and ghrelin responses to a test meal in bulimia nervosa. Biol Psychiatry. 2005; 57(8):926-31.

41. Monteleone P, Santonastaso P, Mauri M, Bellodi L, Erzegovesi S, Fuschino A, et al. Investigation of the serotonin transporter regulatory region polymorphism in bulimia nervosa: relationships to harm avoidance, nutritional parameters, and psychiatric comorbidity. Psychosom Med. 2006; 68(1):99-103.

42. Laessle RG, Beumont PJ, Butow P, Lennerts W, O'Connor M, Pirke KM, et al. A comparison of nutritional management with stress management in the treatment of bulimia nervosa. Br J Psychiatry. 1991; 159(2):250-61.

43. Brambilla F, Draisci A, Peirone A, Brunetta M. Combined cognitive-behavioral, psychopharmacological and nutritional therapy in bulimia nervosa. Neuropsychobiology. 1995; 32(2):68-71.

44. Beumont PJ, Russell JD, Touyz SW, Buckley C, Lowinger $\mathrm{K}$, Talbot $\mathrm{P}$, et al. Intensive nutritional counseling in bulimia nervosa: a role for supplementation with fluoxetine? Aus N Z Psychiatry. 1997; 31(4):514-24.

45. Hsu LK, Rand W, Sullivan S, Liu DW, Mulliken B, McDonagh B, et al. Cognitive therapy, nutritional therapy and their combination in the treatment of bulimia nervosa. Psychol Med. 2001; 31(5):871-9.

46. Sundgot-Borgen J, Rosenvinge JH, Bahr R, Schneider LS. The effect of exercise, cognitive therapy, and nutritional counseling in treating bulimia nervosa. Med Sci Sports Exerc. 2002; 34(2): 190-5.

47. Willard SG, Anding RH, Winstead DK. Nutritional counseling as an adjunct to psychotherapy in bulimia treatment. Psychosomatics. 1983; 24(6): 545-7.

48. Ventura M, Bauer B. Empowerment of women with purging-type bulimia nervosa through nutritional rehabilitation. Eat Weight Disord. 1999; 4(2):55-62.

49. Salvy SJ, McCargar L. Nutritional interventions for individuals with bulimia nervosa. Eat Weight Disord. 2002; 7(4):258-67.

Recebido em: 22/10/2008

Versão final reapresentada em: 23/7/2009 Aprovado em: 4/5/2010 